\title{
Eficacia clínica de la asociación amoxicilina/sulbactam (4:1) en otitis media aguda en niños*
}

JOSÉ COFRÉ G., ANDRÉS ROSENBLÜT R., CECILIA BOREL M., PATRICIA GONZÁLEZ A., ALICIA VÁSQUEZ M., M. TERESA SIRI A. e IRMELI ROINE A.

\section{Clinical efficacy of amoxicillin/sulbactam (4:1) association in acute otitis media in children}

Use of increased amoxicillin dose associated with beta-lactamase inhibitor is a reasonable strategy to counterbalance increasing $S$. pneumoniae and $H$. influenzae resistance in AOM. Aim: To evaluate clinical efficacy and tolerance of amoxicillin/sulbactam in a 4:1 proportion in children with AOM. Methods: In an open, 18 month, treatment study, children with 3-86 months, diagnosed at 2 emergency rooms of Santiago, Chile with AOM by ENT specialist without previous antimicrobial treatment were enrolled. After informed consent a diagnostic tympanocentesis was performed and children were prescribed 50/12,5 mg/kg of amoxicillin/sulbactam, bid for 10 days. Children were followed with clinical exam on days 3,10, and 40 and impedance testing on day 10 and 40 if previously abnormal. The Dagan score (DS) was used for evaluation and criteria for improvement/cure were predefined. Results: 172 children (mean age: 30,9 months, range 4-86) completed the study. Most common microorganisms isolated were S. pneumoniae $40 \%$ (30\% penicillin resistance), Haemophilus $28 \%$ (10.9\% $\beta$-lactamase producers), and Moraxella 4\%. Median DS decreased from 10 at enrollment to 3 on day 3 and 1 on day 10 ( $p<0.001$ ). At day 10 and 40, 48.1/74.1\% were cured, 45.1/18.5\% improved, and 6.8/3.1\% had failed treatment. Diarrhea increased from $6.4 \%$ at enrollment to $32.5 \%$ on day 3 and $8.1 \%$ on day 10 , requiring dietary management only. Two children discontinued treatment due to vomiting one and exanthema another. Conclusions: Amoxicillin/sulbactam at 100/25 mg/kg/ day for 10 days had a clinical efficacy (healing or improvement) of 93.2\% in the treatment of AOM. Transitory diarrhea was common although not severe requiring dietary restriction only.

Key words: Acute otitis media; Etiology; Amoxicillin; Sulbactam; Efficacy; Safety.

Palabras claves: Otitis media aguda; Etiología; Amoxicilina; Sulbactam; Eficacia; Seguridad.

\section{Introducción}

La otitis media aguda (OMA) es una de las enfermedades más comunes en lactantes y preescolares, $80 \%$ de los niños bajo 2 años de edad experimenta episodios de OMA, mientras que $50 \%$ experimenta 3 o más episodios ${ }^{1}$.

Universalmente Streptococcus pneumoniae,
Haemophilus influenzae y Moraxella catarrhalis, están presentes en el fluido del oído medio en alrededor del $70 \%$ de los casos de OMA. En nuestro medio, A. Rosenblüt et al, actualizaron recientemente esta información etiológica ${ }^{2}$.

Amoxicilina continúa siendo el fármaco de primera elección para la otitis media aguda no complicada, por su eficacia clínica sobre infec-

Hospital Dr. Sótero Del Río: Servicio de Otorrinolaringología (ARR) y Laboratorio de Microbiología (PGA)

Hospital Luis Calvo Mackenna: Servicio de Pediatría (JCG, AVM, IRA), Unidad de Otorrinolaringología - Servicio de Cirugía (CBM), Laboratorio de Microbiología (MTSA).

Recibido: 3 diciembre 2003

Aceptado: 24 marzo 2004

* Estudio diseñado por los autores y financiado por Laboratorio Bagó de Chile S.A. 
ciones causadas por S. pneumoniae y sobre $H$. influenzae no productores de $\beta$-lactamasa ${ }^{3,4}$.

El surgimiento mundial y nacional de resistencia de $S$. pneumoniae a penicilina y la existencia conocida de $H$. influenzae y $M$. catarrhalis productores de $\beta$ lactamasas -esta última de escasa prevalencia en OMA en nuestro país ${ }^{2,5,6}$ - ha sido invocado para explicar el fracaso clínico y las recurrencias en el corto plazo, de tratamientos correctamente prescritos y bien cumplidos 7,8 . Hasta $30 \%$ de las cepas de S. pneumoniae recuperadas de infecciones superficiales en pacientes chilenos bajo 5 años de edad, tienen algún grado de resistencia a penicilina: resistencia intermedia (también llamada relativa) o resistencia (resistencia alta $)^{9} . H$. influenzae produce $\beta$-lactamasa tipo TEM-1 en $8 \%^{10}$ hasta $30 \%{ }^{11}$ de las cepas estudiadas en nuestro medio.

Para combatir las OMA causadas por $S$. pneumoniae resistente se ha recomendado empíricamente duplicar las dosis habituales de amoxicilina (de 40-45 mg/kg/día a 80-90 mg/kg/ día) en un afán de superar ampliamente la CIM $_{90}$ de la cepa infectante ${ }^{4}$. El conocimiento farmacocinético establece que en dosis diaria de 45 $\mathrm{mg} / \mathrm{kg} /$ día la concentración de amoxicilina en el fluido del oído medio es de 1 a $6 \mu \mathrm{g} / \mathrm{ml}^{12}$ y en dosis de $90 \mathrm{mg} / \mathrm{kg} /$ día se alcanza a 3 a $8 \mu \mathrm{g} / \mathrm{ml}^{13}$.

La existencia de OMA causadas por $H$. influenzae y $M$. catarrhalis resistentes a amoxicilina ha estimulado a la industria farmacéutica a desarrollar nuevas estrategias de tratamiento y al médico clínico a evaluar estas alternativas terapéuticas. Así se han licenciado cefalosporinas de $2^{\circ}$ y $3^{\circ}$ generación estables a $\beta$-lactamasas del tipo TEM-1 e inhibidores de $\beta$-lactamasas (ácido clavulánico, sulbactam) que conservan la actividad antibacteriana de amoxicilina sobre $H$. influenzae y $M$. catarrhalis, para tratar exitosamente un mayor número de $\mathrm{OMA}^{14}$.

Un inconveniente de las asociaciones de amoxicilina con inhibidores de $\beta$-lactamasas es la producción de diarrea (10-30\%), efecto atribuible fundamentalmente al inhibidor de $\beta$-lactamasa ${ }^{15}$. Un aumento en la dosis diaria de amoxicilina como ha sido propuesto, empleando estas asociaciones, pudiera incrementar este efecto adverso desincentivando su prescripción para el tratamiento de la OMA. Para evitar este inconveniente se han diseñado y ensayado con éxito, fórmulas en que la relación amoxicilina: ácido clavulánico se ha modificado desde 4:1 a 7:1, y recientemente a 14:1, las que aportan mayor dosis de amoxicilina con igual dosis del inhibidor ${ }^{16}$.

Otro aspecto que atenta contra el buen éxito del tratamiento antimicrobiano de la OMA es la poca adherencia al mismo por un alto número de dosis diarias. Así se ha desarrollado el concepto de administrar fármacos dos veces al día (p. ej. axetil cefuroxima, cefprozil) o, una vez al día (p. ej. azitromicina, cefixima), con resultados clíni$\cos$ satisfactorios que han permitido licenciar estos tratamientos ante la F.D.A. de E.U.A. Actualmente se recomienda prescribir amoxicilina y su combinación con ácido clavulánico dos veces al día ${ }^{17}$ basado en el principio farmacodinámico que "un antibiótico $\beta$ lactámico conserva su efecto bactericida siempre que su concentración en el oído medio supere la $\mathrm{CIM}_{90}$ durante al menos 40 a $50 \%$ del lapso de tiempo que media entre dos dosis del mismo" 18,19 . Recientemente R. Dagan et al. demostraron la eficacia clínica/bacteriológica de amoxicilina/ácido clavulánico dosificado cada 12 horas $^{16,20}$, esquema que favorece la adherencia al tratamiento.

En el presente ensayo se emplearon simultáneamente en un mismo producto, tres de las mencionadas estrategias terapéuticas: asociar amoxicilina + sulbactam como inhibidor de $\beta$ lactamasa, elevar las dosis de amoxicilina a 100 $\mathrm{mg} / \mathrm{kg} /$ día y fraccionar su administración diaria en dos tomas (cada 12 horas)

\section{Objetivos}

Primario. Evaluar la eficacia clínica de la asociación amoxicilina/sulbactam en una nueva formulación (cada $5 \mathrm{ml}$ : amoxicilina $1.000 \mathrm{mg}+$ sulbactam $250 \mathrm{mg}$ ), administrando una dosis total diaria de $100 \mathrm{mg} / \mathrm{kg} /$ día, fraccionada cada 12 horas, en el tratamiento de la OMA bacteriana.

Secundario. Evaluar la tolerancia digestiva al medicamento administrado con dicho régimen posológico.

\section{Diseño del estudio}

Estudio abierto, no comparativo, multicéntrico. Se adoptaron los criterios recomendados por Infectious Diseases Society of America en $1992^{21}$ para la evaluación de antimicrobianos en otitis media aguda: definición de OMA, edad de los pacientes, tamaño de muestra, criterios de inclusión y exclusión, ejecución de estudio etiológico, forma de evaluación de los pacientes durante el seguimiento, criterios de curación, mejoría y fracaso, plazos de seguimiento y realización de impedanciometría al término del estudio. Sólo no se practicó la metodología del doble ciego por no emplearse en este estudio un fármaco comparador. 


\section{Pacientes y Métodos}

Pacientes portadores de OMA presuntamente bacteriana, consultantes en las unidades de emergencia de los hospitales Dr Sótero Del Río (HSDR) y Luis Calvo Mackenna (HLCM).

Se estimó necesario estudiar un mínimo de 150 pacientes evaluables en un período no inferior a 12 meses, con el objeto de tener debidamente representadas las dos principales etiologías ( $60 \mathrm{~S}$. pneumoniae y $\sim 48 \mathrm{H}$. influenzae) y sus esperables patrones de susceptibilidad y resistencia in vitro (S. pneumoniae: $30 \%$ de cepas resistentes a penicilina, $H$. influenzae: 20 a $30 \%$ de cepas productoras de $\beta$-lactamasa).

Criterios de inclusión. Pacientes de ambos sexos, entre 3 meses y 6 años de edad (peso máximo $20 \mathrm{~kg}$ ), con signos y síntomas de OMA con evolución no mayor a 4 días. El diagnóstico de OMA se basó en la presencia de otalgia, fiebre, irritabilidad, asociadas a una o más de las siguientes modificaciones de la membrana timpánica: abombamiento, eritema generalizado, disminución del reflejo luminoso de la misma.

Criterios de exclusión. Pacientes con las siguientes condiciones: otorrea de más de 24 horas de evolución, uso de antimicrobianos en los 7 días previos, presencia de tubos de ventilación o antecedente de otra cirugía otológica, signos de cronicidad como perforación antigua de la membrana timpánica u otitis media colesteatomatosa, malformaciones orofaciales, inmunodeficiencias congénitas o adquiridas, desnutrición severa (grado III), enfermedad gastrointestinal crónica o recurrente, antecedentes de hipersensibilidad a $\beta$-lactámicos, necesidad de recibir simultáneamente otros antimicrobianos o terapia antiinflamatoria sistémica.

Tratamiento. Suspensión de amoxicilina/ sulbactam (4:1), en una concentración de amoxicilina $200 \mathrm{mg} / \mathrm{ml}$ y sulbactam $50 \mathrm{mg} / \mathrm{ml}$, la que fue proporcionada por la casa matriz del laboratorio fabricante, Bagó, Buenos Aires, Argentina. Dosificación calculada en base a amoxicilina: $100 \mathrm{mg} / \mathrm{kg} /$ día, fraccionada en 2 tomas (c/12 horas), durante 10 días. La suspensión fue preparada por una enfermera del proyecto de manera de evitar errores de dilución. Se instruyó a los adultos responsables del cumplimiento del tratamiento del paciente para mantener el medicamento entre 4 y $8^{\circ} \mathrm{C}$ y agitar bien cada vez, antes de usar.

\section{Controles}

Visita inicial (Día 1).

Evaluación pediátrica: Registro de datos biomédicos generales, tipo de alimentación en lactantes (natural o artificial), concurrencia a guardería infantil o colegio; episodios previos de OMA, antecedente personal de OMA recurrente o en padres y/o hermanos, alteraciones del tránsito digestivo e irritación glúteogenital previas al inicio del tratamiento.

Evaluación otorrinológica: incluyó otomicroscopia, medición basal del score de Dagan y estudio etiológico.

Evaluación oto-microscópica. Los hallazgos se clasificaron, consignando por separado cada oído, de acuerdo con la escala siguiente: OTO-0: Membrana timpánica (MT) normal; OTO-1: MT congestiva con visualización de la apófisis corta y mango del martillo; OTO-2: MT marcadamente congestiva con pérdida de la visualización de la apófisis corta del martillo (tímpano bombée); OTO-3: Miringitis bulosa; OTO-4: MT perforada y supurando.

Score de Dagan. Se empleó para medir la intensidad de las manifestaciones clínicas de la $\mathrm{OMA}^{22}$. En breve, el score de Dagan emplea 5 parámetros clínico/otológicos: fiebre, irritabilidad, tracción espontánea del pabellón auricular por el paciente, congestión timpánica y abombamiento del tímpano, categorizados c/u de 0 a 3 (= ausente, leve, moderado, severo), con puntaje máximo asignable de 15 . Se catalogó de OMA leve a score < 3; moderada el score 3-7 y severa al score 8-15.

La irritabilidad/otalgia: fue evaluada por el investigador al momento de la primera consulta y de allí en adelante, en forma diaria por el apoderado, empleando la escala de figuras faciales elaborada por la International Association for Study of Pain-IASP, con graduación de 1 a 9 según se $\operatorname{adjunta}^{23}$ (Figura 1).

El familiar o apoderado recibió un formulario para el registro diario de la tolerancia del fármaco: aparición de vómitos, frecuencia y carácter de deposiciones (normales, blandas, líquidas), presencia de erupciones cutáneas y/o irritación glúteogenital, anotaciones que debía completar diariamente y exhibir en los controles.

Para el tratamiento sintomático de la fiebre, otalgia y cefalea se proporcionó paracetamol en suspensión o comprimidos, evitando el empleo de anti-inflamatorios.

Estudio etiológico. Se efectuó obtención del fluido del oído medio luego de esterilizar el conducto auditivo externo con alcohol de 70 grados y procurando siempre no entrar en contacto con estas paredes al momento de puncionar la membrana timpánica. Se efectuó miringotomía sobre el tímpano más afectado, empleando como anes- 


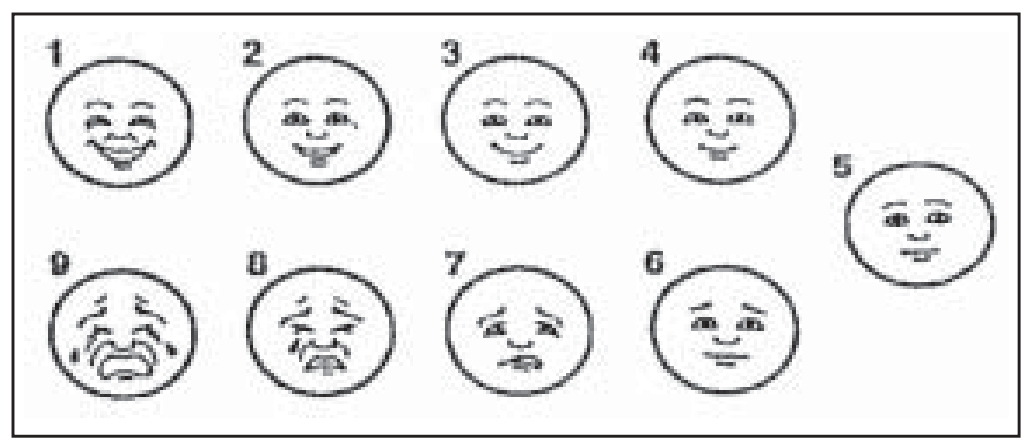

Figura 1. Modelo de figuras faciales para evaluación de dolor en niños. tésico local una solución de prilocaína / lidocaína $5 \%$ en partes iguales $(\text { EMLA } ®)^{24}$. El contenido de oído medio se envió a cultivo bacteriano y estudio de susceptibilidad in vitro.

\section{Seguimiento}

Visita 2 (Día 3 ó 4): Se evaluó el cumplimiento del tratamiento midiendo el volumen consumido del fármaco, la tolerancia al medicamento registrada por los apoderados en formulario adhoc y la eficacia clínica momentánea mediante el control oto-microscópico bilateral. Se programó efectuar nueva miringotomía, con fines terapéuticos y para certificar la curación bacteriológica, sólo en caso de persistir compromiso del estado general o dolor intolerable.

Visita 3 (Días 10 u 11): Se evaluó nuevamente el cumplimiento del tratamiento y su tolerancia. Se efectuó evaluación de la eficacia clínica global del tratamiento antimicrobiano terminado con la inspección oto-microscópica bilateral y se ejecutó impedanciometría para detectar la presencia residual de efusión en el oído medio, mediante un equipo AUDIOTEST 4621 $®$, GAES, España (HSDR) o AUDIOMETER MT 10®, Interacoustics, Dinamarca (HLCM).

Visita 4 (Día $40+2$ ): En aquellos pacientes que no hubieran normalizado el examen otomicroscópico o tuvieran anormalidades en la impedanciometría, se efectuó un nuevo examen oto-microscópico bilateral + impedanciometría, evaluación de nuevos síntomas no relacionados con la enfermedad inicial ni con el uso de la medicación, el requerimiento de otros medicamentos que hubieran sido necesarios para la mejoría de los síntomas y la eficacia global del tratamiento efectuado.

\section{Evaluación de la eficacia clínica}

Eficacia clínica momentánea (Visita 2). Se consideró respuesta favorable el score de Dagan $0-2$ y respuesta insuficiente un score de 3 puntos o más.
Eficacia clínica global (Visita 3 y eventualmente Visita 4). Se clasificó según la siguiente escala:

- Curación. Otomicroscopia: OTO-0 e impedanciometría normal (ausencia de fluido en oído medio).

- Mejoría. Otomicroscopia: OTO-1 e impedanciometría anormal (persistencia de fluido en oído medio).

- Fracaso. Otomicroscopia: OTO-2 a OTO-4 e impedanciometría anormal

Procedimientos microbiológicos. El fluido de oído medio obtenido fue sembrado antes de 15 minutos en agar sangre de cordero y en agar chocolate e incubado en atmósfera de $\mathrm{CO}_{2}$ al $10 \%$. La identificación de especie fue efectuada según técnicas antes descritas en publicación previa de uno de los autores (ARR) $)^{2}$. A las cepas de $S$. pneumoniae se les efectuó determinación de CIM mediante epsilometría (E-test $\left.{ }^{\circledR}\right)$ para los siguientes antimicrobianos: penicilina $\mathrm{G}$, amoxicilina, cefuroxima axetil, cloranfenicol y mediante técnica de difusión en agar de Kirby-Bauer para cotrimoxazol, empleando los valores de corte recomendados por NCCLS en su versión $2000^{25}$. La actividad in vitro de amoxicilina/ sulbactam en la razón 4:1 se evaluó por técnica de Kirby-Bauer empleando los siguientes criterios: susceptible: > $17 \mathrm{~mm}$, intermedio: 12-16 $\mathrm{mm}$, resistente: $<11 \mathrm{~mm}$, según pauta proporcionada por José María Casellas, Bs Aires, Argentina. A las cepas de $H$. influenzae se les determinó CIM para amoxicilina por E-test $\AA$ según criterios NCCLS $2002^{26}$ y la producción de $\beta$-lactamasa por el test de nitrocefina. Las cepas de $S$. pneumoniae fueron almacenadas a $-70^{\circ} \mathrm{C}$ y transportadas para su posterior serotipificación en medio de Amies con carbón activado.

Serotipificación de S. pneumoniae: Fue efectuada en el laboratorio de Streptococcus, Respiratory Diseases Branch, CDC, Atlanta, E.U.A., empleando sucesivamente seroagrupación por lá- 
tex mediante sueros del Staten Serum Institute de Copenhagen y serotipificación por test de hinchamiento capsular (Neufeld-Quellung test) aplicando sueros elaborados en el CDC de Atlanta.

\section{Evaluación de la tolerancia digestiva}

Se prestó especial atención a la presencia de trastornos digestivos (vómitos, diarrea) previos al inicio del tratamiento, su aparición durante el mismo y su evolución posterior. Se definió como diarrea para estos efectos: la presentación de tres o más deposiciones líquidas en un día, o 2 deposiciones líquidas por día durante dos días consecutivos. Se consideró deposiciones blandas a aquellas con menor consistencia que la habitual para cada paciente, sin llegar a ser líquidas.

\section{Eventos adversos}

La posibilidad de aparición de un evento adverso fue evaluada a lo largo del ensayo, pero en especial durante la etapa de tratamiento activo (visitas 2 y 3), su magnitud y eventual relación causa efecto con el fármaco en estudio. Se utilizaron las pautas establecidas por la OMS para la clasificación de la causalidad del fenómeno con el fármaco administrado, en: segura, probable, posible y remota. Se vigiló en especial la aparición de erupción cutánea y/o eritema glúteo/ genital consignando el día de inicio y duración.

\section{Resguardos éticos}

El presente protocolo fue aprobado por las Comisiones de Ética de los Hospitales Dr. Sótero Del Río y Luis Calvo Mackenna. Se solicitó el consentimiento informado escrito a los padres, apoderados o encargados de cada niño para ser incorporado al protocolo.

\section{Resultados}

En 18 meses (diciembre 2000 - mayo 2002) se reclutaron 182 pacientes; completaron el tratamiento 172, y hubo abandono de 10 (5,4\%), uno por exantema, otro por presentar vómitos frecuentes y 8 voluntariamente, por desistir en el cumplimiento del protocolo. Por institución ello significó: HSDS: 4/123 (3,2\%); HLCM: 6/59 $(10,1 \%)$.

Características de los pacientes. En la Tabla 1 se aprecian las principales variables biomédicas de los pacientes tratados. Cerca de $80 \%$ eran lactantes o preescolares y $20 \%$ tenían bajo 1 año de edad (dato no tabulado); la edad promedio fue de: 30,9 meses (rango 4-86 m), sólo 9,3\% tenían sobre 5 años. Tenían OMA previa, OMA recurrente y familiares con OMA recurrente el $47,6,27,3$ y $34,3 \%$ respectivamente. Sobre $2 / 3$ de los pacientes bajo un año de edad recibían lactancia natural al momento del estudio. Asistían a guardería infantil/colegio $31 / 53(58,4 \%)$ de los pacientes del HLCM versus 36/119 (30,2\%) del HSDR $(\mathrm{p}<0,0001)$

La magnitud de la OMA al ingreso al estudio fue importante, con francos signos inflamatorios del tímpano como se analiza más adelante, $18 \%$ tenían otorrea al consultar (Tabla 6).

Etiología de la otitis media aguda. Se identificó la etiología en 153 de 182 (84\%) pacientes reclutados (incluyendo 10 que abandonaron el protocolo) correspondiendo a: $S$. pneumoniae 40,5\% (n: 73), H. influenzae 23,3\% (n: 42), $M$. catarrhalis y $S$. pyogenes, ambos con una frecuencia de 4,4\% (n: 8), otras especies del género Haemophilus 4,4\% (n: 8). Es destacable la coinfección por dos especies en 5,0 \% (n: 9) de

Tabla 1. Variables biomédicas de 172 pacientes con OMA

\begin{tabular}{lcc}
\hline Edad & $\mathbf{N}^{\mathbf{0}}$ & $(\boldsymbol{\%})$ \\
\hline $2-24$ meses & 82 & 47,6 \\
$25-48$ meses & 53 & 30,8 \\
$49-60$ & 19 & 11,0 \\
$>60$ & 16 & 9,3 \\
No precisada & 2 & 1,1 \\
Varón / mujer & $91 / 81$ & $52,9 / 47,1$ \\
OMA previa / recurrente & $82 / 47$ & $47,6 / 27,3$ \\
Antecedente familiar de OMA recurrente & 59 & 34,3 \\
Lactancia natural actual & $25 / 36$ & 69,4 \\
Asiste a guardería & 67 & 38,9 \\
OMA actual bilateral & 48 & $(27,9 \%)$ \\
\hline
\end{tabular}

*Considera sólo lactantes < 12 meses. 
Tabla 2. Hallazgos bacteriológicos en pacientes consultantes por otitis media aguda (Cultivo de oído medio por punción timpánica)

\begin{tabular}{|c|c|c|c|c|c|c|}
\hline \multirow[t]{2}{*}{ Especie } & \multicolumn{2}{|c|}{ SDR } & \multicolumn{2}{|c|}{ HLCM } & \multicolumn{2}{|c|}{ Total } \\
\hline & $\mathbf{N}^{\circ}$ & $(\%)$ & $\mathbf{N}^{\circ}$ & $(\%)$ & $\mathbf{N}^{\circ}$ & $(\%)$ \\
\hline Streptococcus pneumoniae & 51 & $(41,4)$ & 22 & $(38,5)$ & 73 & $(40,5)$ \\
\hline Haemophilus influenzae & 33 & $(26,8)$ & 9 & $(15,7)$ & 42 & $(23,3)$ \\
\hline Haemophilus sp & 1 & $(0,8)$ & 7 & $(12,2)$ & 8 & $(4,4)$ \\
\hline Streptococcus pyogenes & 7 & $(5,6)$ & 1 & $(1,7)$ & 8 & $(4,4)$ \\
\hline Moraxella catarrhalis & 7 & $(5,6)$ & 1 & $(1,7)$ & 8 & $(4,4)$ \\
\hline Otros & 1 & $(0,8)$ & 3 & $(5,1)$ & 4 & $(2,2)$ \\
\hline Cultivo mixto $*$ & 9 & $(7,3)$ & 0 & & 9 & $(5,0)$ \\
\hline Subtotal (+) & 109 & $(88,6)$ & 43 & $(75,4)$ & 152 & $(84,4)$ \\
\hline Cultivo (-) & 12 & $(9,7)$ & 13 & $(22,8)$ & 25 & $(13,8)$ \\
\hline Contaminado & 2 & $(1,6)$ & 1 & $(1,7)$ & 3 & $(1,6)$ \\
\hline Total & 123 & & 57 & & 180 & \\
\hline
\end{tabular}

*S. pneumoniae + H. influenzae (n: 5); S. pneumoniae + otros (n: 2); H. influenzae + otros (n: 2).

Tabla 3. Susceptibilidad in vitro de S.pneumoniae a penicilina determinada por epsilometría $\left(\mathbf{E}-\text { test }^{\circledR}\right)^{*}$

\begin{tabular}{|c|c|c|c|c|}
\hline Hospital & $\mathbf{N}^{\circ}$ & $\begin{array}{c}\text { Susceptible } \\
\% \quad\left(\mathbf{N}^{\circ}\right)\end{array}$ & $\begin{array}{cl}\text { Resistencia intermedia } \\
\% & \left(\mathbf{N}^{\circ}\right)\end{array}$ & $\begin{array}{c}\text { Resistente } \\
\% \quad\left(\mathbf{N}^{\circ}\right)\end{array}$ \\
\hline HSDR & 58 & $74,1 \quad(43)^{*}$ & $17,2 \quad(10)$ & $\begin{array}{c}8,6(5) \\
\text { rango } 2-6 \mu \mathrm{g} / \mathrm{ml}\end{array}$ \\
\hline HLCM & 22 & $59,0 \quad(13)^{*}$ & $31,8 \quad(7)$ & $9,0 \quad(2)$ \\
\hline Total & 80 & $70,0 \quad(56)$ & $21,2 \quad(17)$ & $8,7 \quad(7)$ \\
\hline
\end{tabular}

Valores NCCLS 2001: (CIM $\mu \mathrm{g} / \mathrm{ml})$

Susceptible: <0,06; resistencia intermedia: 0,12-1,0; resistencia: > 2

* p 0,297

los pacientes (Tabla 2). Las etiologías de los episodios en 10 pacientes que abandonaron el protocolo fueron: S. pneumoniae (n: 7), Haemophilus sp (n: 2) y cultivo negativo (n: 1).

Serotipos. En un total de 80 cepas de $S$. pneumoniae estudiadas se identificaron 23 serotipos, los principales fueron: $19 \mathrm{~F}(20 \%), 14$ $(13,7 \%), 19$ A $(10 \%)$ y $3(5 \%)$; otros serotipos detectados fueron: $1,3 \mathrm{~B}, 4,5,6 \mathrm{~A}, 6 \mathrm{~B}, 7 \mathrm{~F}, 8,9$ $\mathrm{V}, 11 \mathrm{~A}, 12 \mathrm{~F}, 15 \mathrm{~B}, 18 \mathrm{C}, 22 \mathrm{~F}, 23 \mathrm{~B}, 23 \mathrm{~F}, 24 \mathrm{~F}$, 34 y 35 B.

\section{Susceptibilidad antimicrobiana}

Streptococcus pneumoniae. La resistencia global a penicilina en las 80 cepas recuperadas por timpanocentesis fue de $29,9 \%$, mayor en HLCM que en HSDR (40,8 vs 25,8 \%), pero sin significación estadística (p 0,297), diferencia determinada por la frecuencia de resistencia intermedia en una y otra institución (31,8\% en HLCM versus 17,2\% en HSDR) (Tabla 3). En los 73 pacientes que completaron el protocolo, la resistencia global fue de $27,7 \%$ (intermedia y resistencia en
18,0 y $9,7 \%$ respectivamente). No se detectó resistencia in vitro a amoxicilina (CIMs en rango 0,016-2,0 $\mu \mathrm{g} / \mathrm{ml}$ ) ni a la combinación amoxicilina/ sulbactam. Se observó 47,0\% (15/67) de resistencia a cotrimoxazol $(32 / 68), 10,1 \%$ de resistencia intermedia a cefuroxima axetil y $1,2 \%$ de resistencia a cloranfenicol.

La frecuencia de resistencia en S. pneumoniae varió según edad: $37,7 \%$ en lactantes, $27,2 \%$ entre 2 y 4 años, $8,3 \%$ sobre 4 años, sin alcanzar significación estadística (p: $\left.0,134, \chi^{2}: 4,023\right)$. No se estableció relación entre resistencia antimicrobiana y asistencia a guardería infantil/colegio en $S$. pneumoniae: $5 / 26$ entre asistentes versus $16 / 49$ entre los no asistentes $\left(\chi^{2}: 0,925 ; \mathrm{p}: 0,336\right)$.

Haemophilus spp y Moraxella sp. Se observó globalmente $20,6 \%$ de producción de $\beta$ lactamasa, sólo 10,9\% en el género Haemophilus y $87,5 \%$ en Moraxella sp (Tabla 4). Todas las cepas no productoras de $\beta$-lactamasa tuvieron CIM para amoxicilina en rango de susceptibilidad. De 58 cepas evaluadas in vitro ante amoxicilina/sulbactam, $54(93,1 \%)$ fueron sus- 
ceptibles, en ninguna de las 4 cepas resistentes se detectó producción de $\beta$-lactamasa. Cloranfenicol fue activo sobre 56/58 cepas $(94,8 \%)$ detectándose una cepa con resistencia intermedia (CIM 4 $\mu \mathrm{g} / \mathrm{ml})$ y otra con resistencia $(\mathrm{CIM}>256 \mu \mathrm{g} / \mathrm{ml})$. Cefuroxima fue activa en 40/42 $(95,2 \%)$ cepas evaluadas con CIMs entre 0,064 y $1,0 \mathrm{mg} / \mathrm{ml} ; 2$ cepas tuvieron CIM > $256 \mu \mathrm{g} / \mathrm{ml}$. Hubo $30,0 \%$ $(15 / 50)$ de resistencia a cotrimoxazol.

\section{Eficacia momentánea}

Fue favorable a los 3 días de tratamiento en algo más de $40 \%$ de los pacientes (Tabla 5). No se observó una mayor recuperación de los pacientes con OMA debida a Haemophilus spp y Moraxella sp que en las causadas por $S$. pneumoniae.

\section{Evolución clínica de los pacientes}

Se observó una paulatina disminución de la anormalidad otológica al examen microscópico (Tabla 6, Figura 2). Al ingreso 62,2\% tenían tímpano abombado (OTO-2) y 36,6\% tenían una importante inflamación timpánica (OTO-3-4). En la Visita 2, 56,3\% habían reducido su anormalidad otológica al mínimo (OTO-1), y 16,8\% a cero (OTO-0), porcentajes que en la Visita 3 eran de 36,0 y $51,1 \%$ respectivamente. Al finalizar el tratamiento (Visita 3), sólo 8,1\% mantenían inflamación timpánica significativa (OTO-2-4), cifra que se mantuvo estable $(11,2 \%)$ en el grupo con seguimiento a 40 días. Se observaron 4 pacientes con reagudización de síntomas intratratamiento: 2 con OMA causada por $H$. influenzae

Tabla 4. Frecuencia de producción de $\beta$-lactamasa por Haemophilus sp y Moraxella sp

\begin{tabular}{lcccc}
\hline \multicolumn{1}{c}{ Especie } & \multicolumn{3}{c}{$\boldsymbol{\beta}$-lactamasa $(+)$} & \multicolumn{2}{c}{$\begin{array}{c}\boldsymbol{\beta} \text {-lactamasa } \\
\boldsymbol{\%}\end{array}$} & $\boldsymbol{\%}$ & $\left(\mathbf{N}^{\circ}\right)$ & $\left(\mathbf{N}^{\circ}\right)$ \\
\hline Haemophilus spp (n: 55) & 10,9 & 6 & 89,0 & 49 \\
Moraxella sp (n: 8) & 87,5 & 7 & 12,5 & 1 \\
Global (n: 63) & 20,6 & 13 & 79,3 & 50 \\
\hline
\end{tabular}

Tabla 5. Eficacia clínica momentánea (día 3-4) global y según etiología de amoxicilina/sulbactam 4:1

\begin{tabular}{|c|c|c|c|c|c|c|}
\hline & \multicolumn{2}{|c|}{ Global } & \multicolumn{2}{|c|}{ S. pneumoniae } & \multicolumn{2}{|c|}{ Haemophilus spp y Moraxella sp } \\
\hline & $\%$ & $\mathbf{N}^{\circ}$ & & $(\%)$ & $\mathbf{N}^{\circ}$ & $(\%)$ \\
\hline Total & 172 & & 65 & & 63 & \\
\hline Favorable * & 75 & $(43,6)$ & 23 & $(35,4)^{1}$ & 25 & $(39,7)^{1}$ \\
\hline Insuficiente $* *$ & 97 & $(56,4)$ & 42 & $(64,6)$ & 38 & $(60,3)$ \\
\hline
\end{tabular}

Score de Dagan * $0-2 ; * * 3$ ó más

${ }^{1} \mathrm{p}=0,45$

Tabla 6. Evolución oto microscópica de la otitis media aguda desde el ingreso hasta fines del estudio

\begin{tabular}{|c|c|c|c|c|c|c|c|c|}
\hline \multirow[b]{2}{*}{ Intensidad* } & \multicolumn{2}{|c|}{$\begin{array}{c}\text { Ingreso } \\
\text { Día } 0\end{array}$} & \multicolumn{2}{|r|}{$\begin{array}{l}\text { Visita } 2 \\
\text { Día 3-4 }\end{array}$} & \multicolumn{2}{|c|}{$\begin{array}{c}\text { Visita } 3 \\
\text { Día 10-11 }\end{array}$} & \multicolumn{2}{|c|}{$\begin{array}{c}\text { Visita } 4 \\
\text { Día } 40+2\end{array}$} \\
\hline & $\mathbf{N}^{\circ}$ & (\%) & $\mathbf{N}^{\circ}$ & (\%) & & $(\%)$ & $\mathbf{N}^{\circ}$ & $(\%)$ \\
\hline OTO - 4 & 31 & $(18,0)$ & 14 & $(8,1)$ & 5 & $(2,9)$ & 3 & $(4,8)$ \\
\hline OTO - 3 & 32 & $(18,6)$ & - & & - & & - & \\
\hline OTO - 2 & 107 & $(62,2)$ & 30 & $(17,4)$ & 9 & $(5,2)$ & 4 & $(6,4)$ \\
\hline OTO - 1 & - & & 97 & $(56,3)$ & 62 & $(36,0)$ & 19 & $(30,6)$ \\
\hline OTO - 0 & - & & 29 & $(16,8)$ & 88 & $(51,1)$ & 36 & $(58,0)$ \\
\hline Sin control & - & & - & & 6 & & - & \\
\hline Total & 170 & (100) & 170 & (100) & 170 & (100) & 62 & (100) \\
\hline
\end{tabular}

* Definiciones en Pacientes y Métodos

p día 3-4 vs ingreso $<0,001$

$\mathrm{p}$ día 10 vs ingreso $<0,001$

p día 40 vs ingreso $<0,001$. 


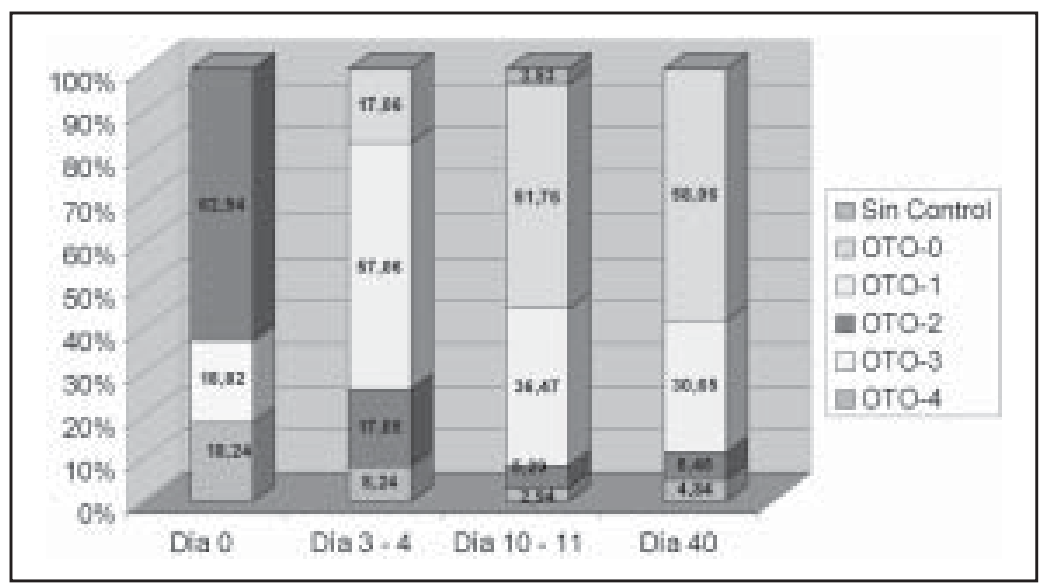

Figura 2. Evolución oto microscópica de la otitis media aguda desde el ingreso hasta fines del estudio.

(edad 10 y 28 meses), 1 por S. pneumoniae (edad 8 meses) y 1 de etiología no precisada (edad 65 meses).

\section{Eficacia clínica global}

Corroborando la evolución clínica otológica con rápida tendencia a la resolución, 93,2\% de los pacientes tuvieron curación o mejoría al término de 10 días de tratamiento con amoxicilina/ sulbactam 4:1 (Tabla 7), sin diferencia significativa según etiología (entre $S$. pneumoniae y $H$. influenzae). El $44,7 \%$ de los pacientes con $S$. pneumoniae y 40,3\% en caso de Haemophilus/ Moraxella tenían líquido en el oído medio y una mínima inflamación timpánica residual como criterio de mejoría sin curación, al completarse los 10 días de tratamiento antimicrobiano. En el seguimiento a 40 días 21,6\% tuvieron aún anormalidades en la impedanciometría.

Fracasó el tratamiento en 11 pacientes $(6,8 \%)$, cuyas etiología eran: $H$. influenzae (4); S. pneumoniae (3); S. pyogenes (1); $P$. aeruginosa (1); cultivo negativo (2), todas estas cepas eran susceptibles in vitro a amoxicilina/sulbactam, excepto $P$. aeruginosa. No se hizo timpanocentesis para control bacteriológico en este grupo.

\section{Tolerancia digestiva}

Se apreció un transitorio incremento en la frecuencia de evacuaciones y mayor contenido acuoso de las deposiciones, en un importante número de pacientes (6,4\% al ingreso, $65,6 \%$ en el día 3-4), en la mitad de ellos con carácter de diarrea; este efecto se extinguió espontáneamente en la mayoría al día 10-11, momento en que sólo 8,1\% aún tenían deposiciones líquidas (Figura 3). Se insinuó una relación entre aparición de diarrea y edad: 39,2\% en lactantes versus $25,8 \%$ en pacientes sobre dos años, sin alcanzar significación estadística (p: $0,069)$.

\section{Efectos adversos}

Durante el seguimiento diario de los pacientes no se comprobaron reacciones adversas severas; se comprobó intolerancia gástrica en un paciente quien suspendió el tratamiento y un exantema morbiliforme fugaz atribuido al fármaco en ensayo, en otro paciente. Ambos tuvieron carácter leve, sin embargo, sus padres decidieron abandonar el protocolo. No se reportó eritema glúteogenital en los pacientes que presentaron diarrea.

Tabla 7. Resolución clínico/impedanciométrica de la otitis media aguda tratada con amoxicilina/sulbactam (4:1)

\begin{tabular}{|c|c|c|c|c|c|c|c|c|c|c|c|c|}
\hline \multirow[t]{3}{*}{ Resolución } & \multicolumn{4}{|c|}{$\begin{array}{l}\text { Global } \\
\text { (N: 162) }\end{array}$} & \multicolumn{4}{|c|}{$\begin{array}{l}\text { S. pneumoniae } \\
(\mathbf{N}: 67)\end{array}$} & \multicolumn{4}{|c|}{$\begin{array}{c}\text { Haemophilus spp y Moraxella sp } \\
(\mathbf{N}: 62)\end{array}$} \\
\hline & \multicolumn{2}{|c|}{ Día 10} & \multicolumn{2}{|c|}{ Día 40} & \multicolumn{2}{|c|}{ Día 10} & \multicolumn{2}{|c|}{ Día 40} & \multicolumn{2}{|c|}{ Día 10} & \multicolumn{2}{|c|}{ Día 40} \\
\hline & $\mathbf{N}$ & $(\%)$ & $\mathbf{N}$ & $(\%)$ & $\mathbf{N}$ & & $\mathbf{N}$ & $(\%)$ & $\mathbf{N}$ & & & $(\%)$ \\
\hline Curación* & 78 & $(48,1)$ & 120 & $(74,1)$ & 34 & $(50,7)$ & 50 & $(74,6)$ & 33 & $(53,2)$ & 41 & $(66,1)$ \\
\hline Mejoría* & 73 & $(45,1)$ & 30 & $(18,5)$ & 30 & $(44,7)$ & 12 & $(17,9)$ & 25 & $(40,3)$ & 15 & $(24,1)$ \\
\hline Fracaso* & 11 & $(6,8)$ & 5 & $(3,1)$ & 3 & $(4,4)$ & 1 & $(1,4)$ & 4 & $(6,4)$ & 3 & $(4,8)$ \\
\hline No determinado & 8 & $(4,9)$ & 7 & $(4,3)$ & - & 4 & - & 3 & & & & \\
\hline
\end{tabular}

* Definiciones en Pacientes y Métodos 


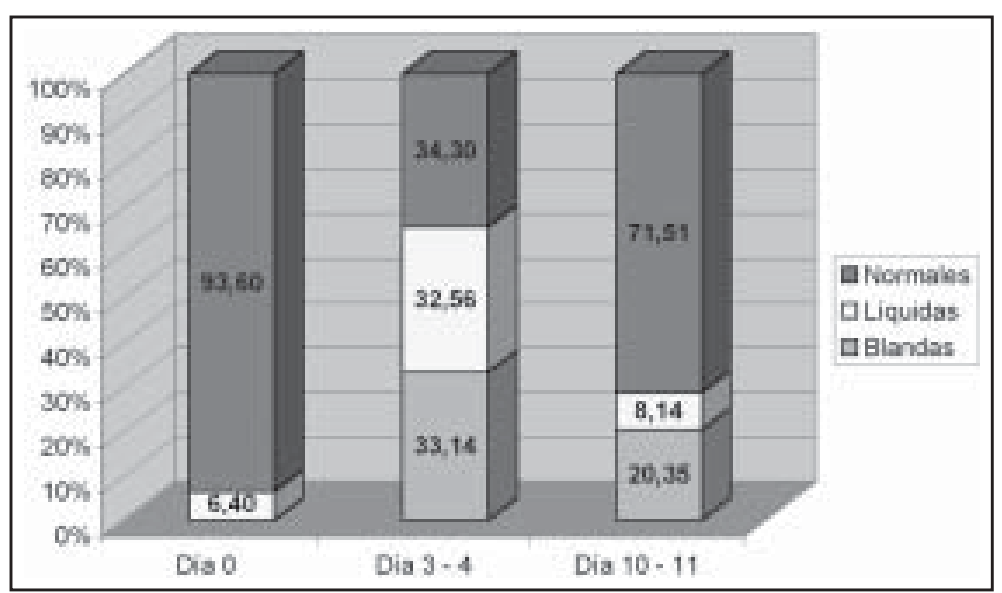

Figura 3. Tolerancia digestiva de la suspensión de amoxicilina/sulbactam (4:1). Frecuencia de deposiciones anormales (blandas o líquidas) antes y durante su administración. Día 3-4 vs día 0: $\mathrm{p}<0,001$; Día 10-11 vs día 3-4: $\mathrm{p}<0,001$.

posibilidad de evaluar esta nueva combinación de antimicrobianos frente a un mayor número de cepas resistentes, lo que podría haber entregado una valiosa información adicional.

En este ensayo corroboramos un concepto trascendente para el médico tratante y que, a pesar de ser conocido por microbiólogos ${ }^{28}$, no ha sido suficientemente difundido: amoxicilina es más activo in vitro que penicilina sobre $S$. pneumoniae; pese a ello aún se continúa haciendo referencia a la susceptibilidad in vitro a penicilina al momento de discutir las indicaciones terapéuticas en OMA, en consideración que los

\section{Discusión}

Las características de la población seleccionada para este estudio, preferentemente lactantes $(47,6 \%)$ y preescolares $(30,8 \%)$, con antecedentes de OMA en $47,6 \%$, OMA recurrente en casi $30 \%$, OMA recurrente en la familia en $1 / 3$ de ellos y asistencia a guardería infantil/colegio en $38,9 \%$, son en términos clínico/epidemiológicos, representativos de la población de niños que consultan por OMA en la Región Metropolitana y posiblemente en otras regiones del país. Dagan y McCracken han discutido recientemente la importancia de la edad -idealmente deben evaluarse niños bajo 2 años de edad-, antecedentes otológicos positivos y asistencia a guarderías infantiles, en la selección de pacientes, al diseñar estudios de evaluación de antimicrobianos en OMA; debe elegirse pacientes que representen cierto grado de dificultad terapéutica ${ }^{27}$.

En 180 pacientes pediátricos con OMA se efectuó timpanocentesis recuperando en $84,4 \%$ de los casos, bacterias del oído medio: en 80 oportunidades $(44,4 \%)$ S. pneumoniae y en 63 pacientes (35\%) Haemophilus o Moraxella spp. Estos hallazgos y el correspondiente estudio de susceptibilidad $(29,9 \%$ de $S$. pneumoniae resistentes a penicilina y $10,9 \%$ de Haemophilus productores de $\beta$-lactamasa) son concordantes con estudios recientes y pretéritos efectuados en el medio nacional ${ }^{2,5,6}$ y eran esperables desde la planificación del estudio como se expresó en Pacientes y Métodos. El haber elegido pacientes sin tratamiento antimicrobiano en curso o reciente, explica el buen rendimiento etiológico obtenido -era una de nuestras metas- pero disminuyó la médicos clínicos emplearán preferentemente amoxicilina. Los valores de corte establecidos por la NCCLS para definir in vitro la resistencia a amoxicilina son más elevados $-4 \mu \mathrm{g} / \mathrm{ml}$ para amoxicilina, según nuevos conocimientos de su farmacocinética versus $0,12 \mu \mathrm{g} / \mathrm{ml}$ para penicilina- ${ }^{26}$, y la eficacia clínica de amoxicilina es mayor que la esperable para "un $30 \%$ de resistencia in vitro a penicilina" ${ }^{4,7,8}$. En las 80 cepas de $S$. pneumoniae estudiadas observamos sistemáticamente CIMs menores para amoxicilina que para penicilina (datos no presentados) con rangos de 0,016 a $2,0 \mu \mathrm{g} /$ $\mathrm{ml}$, sin detectar resistencia in vitro a amoxicilina. La actividad in vitro de cefuroxima fue inferior a la de amoxicilina sobre $S$. pneumoniae, argumento que también debe considerarse al elegir un antibiótico antineumocóccico en general. Cloranfenicol fue sistemáticamente activo, no así cotrimoxazol ante el cual la resistencia afectó a una de cada 2 cepas estudiadas.

Se detectó una amplia dispersión en la distribución de los serotipos, sólo 30\% eran del serogrupo 19F/A y $13,7 \%$ del serotipo 14 , el resto $(56,3 \%)$ se distribuyó en 20 serotipos diferentes. Rosenblüt et al. detectaron 18 serotipos siendo también $19 \mathrm{~F}$ el más frecuente $(15,8 \%)$ en su casuística ${ }^{2}$. Esta distribución difiere de la encontrada por R. Lagos et al, recientemente en nuestro medio para infecciones invasoras, en un grupo etario similar de la Región Metropolitana; en su experiencia predominaron los serotipos 14, 19F y $18 \mathrm{C}$ sumando entre 31,5 y $43,2 \%$ del total según la localización de la infección que se considere $^{29}$. Esta diferencia es importante de valorar cuando se hacen ejercicios de estimación de la cobertura otorgada por vacunas conjugadas para 
enfermedades neumocóccicas en nuestro medio.

Contra las cepas de Haemophilus sp, tanto amoxicilina como amoxicilina/sulbactam, cefuroxima y cloranfenicol tuvieron una actividad in vitro superior a $90 \%$ siendo todas alternativas terapéuticas valederas según nuestra serie.

C. Bluestone describe en su texto clásico: Otitis Media Aguda, que a un mes de terminado el tratamiento de una OMA con amoxicilina, $80 \%$ de los pacientes resuelven totalmente el cuadro y en el $20 \%$ restante persiste una efusión residual, generalmente aséptica ${ }^{30}$. Nuestra meta de alcanzar una eficacia clínica al menos similar a ésta, se cumplió cabalmente; en la otomicroscopia efectuada al ingresar al estudio, todos los pacientes tenían una OMA con intensidad OTO 2-4, sólo 25,5\% presentaban esta magnitud al día 3-4 y $8,1 \%$ al día $10-11$. Por otra parte, la impedanciometría fue normal en 48,1\% al día 10-11 y en $74,1 \%$ al día 40 ; persistió la efusión media en $51,9 \%$ de los casos al $10^{\circ}$ día y en $21,6 \%$ a un mes de finalizar el tratamiento, en concordancia con lo referido por Bluestone ${ }^{30}$.

Es conocido que un número significativo de las OMA cura espontáneamente, especialmente en niños mayores o si se trata de un episodio aislado; si la etiología es $H$. influenzae, esta curación, sin mediar terapia antiinfecciosa, puede alcanzar casi a $50 \%$ y en caso de otitis neumocóccicas, la resolución espontánea es de aproximadamente $20 \%{ }^{31,32}$. Por ello se ha planteado que la eficacia de una terapia anti infecciosa en OMA debiera ser medida en términos de curación bacteriológica (timpanocentesis de control al $3^{\circ}-4^{\circ}$ día de tratamiento) según definieran Howie y Ploussard en $1969^{31}$ y reafirmaran recientemente Dagan et $\mathrm{al}^{22}$ y Dagan y McCracken ${ }^{27}$; este test de eficacia in vivo es actualmente exigible en estudios comparativos para introducir nuevos fármacos al uso clínico. Pero resulta éticamente difícil de sustentar una doble timpanocentesis en nuestro estudio, abierto y no comparativo; J. O. Klein ${ }^{33,34}$ cuestiona la necesidad de efectuar doble punción timpánica para conocer la eficacia de un tratamiento antimicrobiano, toda vez que un número importante de cultivos continúan siendo positivos al $3^{\circ}-4^{\circ}$ día y sin embargo, los pacientes mejoran de su dolencia pocos días después ${ }^{22}$. Por otro lado, en la práctica clínica se recomienda reservar la tímpanocentesis sólo para aquellos casos en que el tratamiento fracasa a las 48-72 horas de iniciado, pensando en el alivio sintomático del paciente y en el beneficio de un diagnóstico etiológico y correspondiente estudio de susceptibilidad ${ }^{33}$. Decidimos entonces adoptar el score de Dagan que correlaciona los hallazgos clínicos y otomicroscópicos al $3^{\circ}-4^{\circ}$ día de tratamiento, con curación bacteriológica a esa altura del tratamiento; en su experiencia, la curación bacteriológica se tradujo en curación clínica en 97\% de los pacientes aunque por otra parte, $37 \%$ de los pacientes con cultivo persistentemente positivo habían curado clínicamente ${ }^{22}$. $\mathrm{Al} 3^{\circ}-4^{\circ}$ día de tratamiento comprobó Dagan que $46 \%$ aún tenían bacterias viables en el fluido del oído medio pero al $10^{\circ}$ día habían curado, resultado atribuible a un efecto bactericida lento o simplemente a la capacidad inmunológica de huésped, como se observa cuando se ha empleado un placebo $^{31,32}$. La respuesta clínica observada en el presente ensayo fue enteramente satisfactoria, con reducción de signos y síntomas de OMA al $3^{\circ}-4^{\circ}$ día en $66,8 \%$ y al $10^{\circ}$ día en $87,1 \%$ de los pacientes.

No se pudo establecer correlación entre respuesta terapéutica y susceptibilidad in vitro al fármaco empleado (datos no presentados); el bajo porcentaje de resistencia in vitro encontrado a la combinación amoxicilina/sulbactam, $0 \%$ a $S$. pneumoniae y $6,9 \%$ a $H$. influenzae, no permitieron un mayor análisis estadístico. Entre los 11 pacientes que no mejoraron, hubo 3 cepas de $S$. pneumoniae, 2 enteramente susceptibles y una de ellas con CIM elevada a penicilina (CIM $2 \mu \mathrm{g}$ / $\mathrm{ml}$ ) pero susceptible a amoxicilina (CIM $0,75 \mu \mathrm{g}$ / $\mathrm{ml}$ ) y 4 cepas de $H$. Influenzae, todas no productoras de $\beta$-lactamasas. Es controversial que exista una estrecha correlación entre susceptibilidad in vitro y resultado clínico en OMA; Dagan et al. documentaron una clara relación entre resistencia in vitro y fracaso bacteriológico al $3^{\circ}-4^{\circ}$ día de tratamiento, aunque la mayoría de sus pacientes mejoró al término del mismo, independientemente de la susceptibilidad in vitro ${ }^{22}$. En otra experiencia, multicéntrica, Hoberman et al no registraron una correlación significativa entre nivel de resistencia in vitro de $S$. pneumoniae a penicilina y resultado clínico, al emplear amoxicilina/ ácido clavulánico en 188 pacientes con otitis neumocóccica ${ }^{35}$. Otros factores intervienen, sin duda, en la evolución del paciente; así: la coexistencia de infección viral dificulta la mejoría ${ }^{36,37}$, la acción del sistema inmune favorece la curación espontánea ${ }^{31,32}$, la alimentación con biberón y la presencia de atopia nasal retardan la mejoría, y por supuesto caben consideraciones de tipo microbiológicas como la especie causal $(S$. pneumoniae determina mayor refractariedad a la curación que Haemophilus sp) y los puntos de corte empleados para asignar resistencia ${ }^{29} \mathrm{o}$ factores farmacológicos. Consideraciones farmacocinéticas: la concentración alcanzada por el fár- 
maco en el fluido del oído medio (COM) ha sido ampliamente variable en ensayos efectuados, como se señalara en la introducción ${ }^{13,14}$, influyendo en ella: la dosis oral administrada, el momento de obtención del fluido del oído medio, el método empleado en la cuantificación del antimicrobiano y el grado de contaminación de la muestra con sangre; incluso se ha reportado una significativa diferencia de COM entre ambos oídos, observada en $86 \%$ de los pacientes en se practicó la doble timpanocentesis ${ }^{13}$. Consideraciones farmacodinámicas: Craig y Andes dedujeron de ensayos publicados con anterioridad que, en una perspectiva farmacodinámica, bacterias con menor CIM son más fáciles de erradicar (COM/CIM y T/CIM favorables), donde T/CIM corresponde al tiempo en que la concentración del antimicrobiano supera en sangre a la CIM, y que por el contrario las cepas más resistentes amenazan el éxito de un tratamiento (COM/CIM y T/CIM desfavorables $)^{18}$.

Desde el punto de vista costo/beneficio, se justifica aún mantener en la comunidad chilena la recomendación de amoxicilina como terapia de primera elección para OMA, considerando su excelente actividad anti neumocóccica (100\% de susceptibilidad in vitro), que las cepas resistentes a amoxicilina representaron sólo 10,9\% de los Haemophilus y que las cepas de Moraxella, si bien en su mayoría eran productoras de $\beta$ lactamasa, fueron minoritarias en la génesis de OMA ( $8 \%$ en nuestra casuística). Ante el fracaso clínico de amoxicilina, en pacientes "vírgenes a uso de $\beta$-lactámicos", las combinaciones de amoxicilina e inhibidores de $\beta$-lactamasas representan una alternativa de segunda instancia terapéutica. Hay circunstancias donde pudiera anticiparse una mala respuesta a amoxicilina: su uso reciente $(<3-4$ semanas) y condiciones de hacinamiento, asistencia a guarderías infantiles, (o hermanos mayores asistentes a guarderías infantiles), dado que la cohabitación con lactantes y preescolares que portan en su faringe cepas resistentes de $S$. pneumoniae y de $H$. influenzae (lo que sucede a diario en salas cunas/jardines infantiles) ha incrementado el porcentaje de fracasos primarios de amoxicilina en el tratamiento de la OMA; en estas situaciones las asociaciones con inhibidores de $\beta$-lactamasas auguran un mayor éxito terapéutico.

Mansilla et $\mathrm{al}^{38}$ demostraron la eficacia clínica de la administración conjunta de amoxicilina y sulbactam en dosis de $50 \mathrm{mg}$ por $\mathrm{kg} /$ día de cada una en el tratamiento de la OMA, en un estudio preliminar que incluyó 58 niños, a los cuales la dosis total diaria les fue administrada fraccionada cada 12 horas. Posteriormente, en un estudio multicéntrico realizado en distintos centros otorrinológicos de Argentina, se incorporó a 222 niños con $\mathrm{OMA}^{39}$. La eficacia clínica obtenida con este esquema de dosificación fue concordante con los datos existentes para la administración cada 8 horas. Ambos ensayos carecieron de estudio etiológico de manera que su alcance es limitado. Nuestros hallazgos microbiológicos permiten extrapolar los resultados exitosos a la población pediátrica general, a la vez que reafirman la información epidemiológica/microbiológica descrita recientemente por uno de los autores ${ }^{2}$.

La emergencia de $S$. pneumoniae resistente a penicilina estimuló la duplicación de la dosis diaria de amoxicilina y en consecuencia, el diseño de asociaciones con inhibidores de $\beta$-lactámicos conteniendo mayor proporción de amoxicilina, para evitar así su intolerancia digestiva. A la luz de recientes definiciones sobre puntos de corte para resistencia, la concentración de amoxicilina en el fluido de oído medio después de administrar esta doble dosis, es teóricamente suficiente para inhibir el crecimiento de cepas de $S$. pneumoniae con resistencia intermedia y algunas con resistencia, aunque no tiene efecto bactericida sobre especies productoras de $\beta$-lactamasas. Preocupante es incrementar la dosificación diaria y la concentración de amoxicilina en preparados para uso pediátrico, por su modulación sobre la microflora intestinal. A manera de ejemplo, se ha definido que no es recomendable superar los 10 $\mathrm{mg} / \mathrm{kg} /$ día de ácido clavulánico para evitar mayor incidencia de intolerancia digestiva ${ }^{4}$. Por eso, otro de los objetivos del estudio fue evaluar la tolerancia digestiva de este preparado farmacéutico que contiene una alta concentración de amoxicilina $(200 \mathrm{mg} / \mathrm{ml})$. La intolerancia gástrica e intestinal de las asociaciones orales de $\beta$ lactámicos con inhibidores de $\beta$-lactamasas es atribuible en parte al $\beta$-lactámico ${ }^{15,40}$; la adición del inhibidor incrementa la frecuencia de esta intolerancia. Los reportes originales sobre tolerancia digestiva de ácido clavulánico y de sulbactam señalaban una frecuencia de 3-5\% de efecto diarreogénico, sin hacer distinción entre adultos y población infantil ${ }^{15}$; sin embargo, posteriores observaciones en niños con OMA, tratada con amoxicilina/ácido clavulánico en dosis de $40 \mathrm{mg} / \mathrm{kg} / \mathrm{día}$ de amoxicilina, incrementaron este porcentaje hasta $\sim 30 \%{ }^{41,42}$. Una evaluación de la combinación amoxicilina/ácido clavulánico en relación 7:1, efectuada en E.U.A., concluyó que $17,8 \%$ de los pacientes tuvieron deposiciones blandas, 14,2\% líquidas, 8,2\% dermatitis del pañal y $3,9 \%$ vómitos; en forma intrigante, la eva- 
luación simultánea efectuada en Europa describió deposiciones blandas en $1,6 \%$, diarrea en $2,2 \%$, y vómitos en $1,7 \%{ }^{35}$. En nuestro ensayo se practicó un seguimiento acucioso de la tolerancia digestiva, observándose un efecto diarreogénico mientras el fármaco estaba siendo aplicado, en $1 / 3$ de los pacientes (similar a lo antes descrito con dosis bajas de amoxicilina), fenómeno que fue bien tolerado y remitió espontáneamente sin mediar indicaciones terapéuticas, aunque persistió hasta el término del mismo en $8,1 \%$ de los pacientes, sugiriendo una suerte de "acostumbramiento" a la disbacteriosis. No se observó una mayor susceptibilidad en lactantes que en niños mayores. La literatura médica indica que la mayor parte de los pacientes que experimentan esta intolerancia mejoran al suspender la administración del fármaco, en nuestra experiencia esta recuperación sucedió intra-tratamiento, reacción que ya había sido descrita con anterioridad ${ }^{40}$.

\section{Conclusión}

Este es el primer estudio pediátrico de eficacia clínica de la asociación amoxicilina/sulbactam en una relación 4:1 sobre la OMA, con conocimiento acabado de su etiología y susceptibilidad in vitro de los agentes causales. Sobre $90 \%$ de los pacientes tuvieron curación o mejoría al término de 10 días de tratamiento. Los resultados avalan su futuro empleo terapéutico exitoso en OMA del lactante y niño mayor. La dosificación de 100 $\mathrm{mg} / \mathrm{kg} / \mathrm{día}$ de amoxicilina y $25 \mathrm{mg} / \mathrm{kg} / \mathrm{día}$ de sulbactam, y su presentación en suspensión de $1.000 \mathrm{mg}$ de amoxicilina y $250 \mathrm{mg}$ de sulbactam cada $5 \mathrm{ml}$, tuvieron una tolerancia digestiva similar a la antes descrita para otras combinaciones de $\beta$-lactámicos orales con inhibidores de $\beta$ lactamasas.

\section{Agradecimiento}

A QF Jaime Inostroza S. por la serotipificación de las cepas de $S$. pneumoniae.

\section{Resumen}

El empleo de dosis elevadas de amoxicilina asociada a inhibidores de $\beta$-lactamasas es una estrategia razonable para eludir la creciente resistencia de $S$. pneumoniae y $H$. influenzae en otitis media aguda (OMA). Objetivo: Evaluar la eficacia clínica y tolerancia de amoxicilina/sulbactam en una combinación 4:1 en niños con OMA. Métodos: en un estudio abierto, no comparativo, efectuado durante 18 meses, se enrolaron niños entre 3 y 86 meses de edad, consultantes en 2 unidades de emergencia de Santiago, Chile, con OMA diagnosticada por otorrinolaringólogo, sin tratamiento antimicrobiano previo. Una vez solicitado el consentimiento informado se les efectuó una timpanocentesis con fines diagnósticos indicándose luego amoxicilina/sulbactam 50/12,5 mg/ $\mathrm{kg}$ dos veces al día durante 10 días. Se efectuó seguimiento con examen clínico en los días 3, 10 y 40 e impedanciometria en el día 10 y 40 si la anterior era anormal. Se empleó el score de Dagan (SD) como evaluación y los criterios de mejoría/curación fueron predefinidos. Resultados: 172 niños (edad promedio: 30,9 meses, rango 4-86) completaron el estudio. Los principales microorganismos aislados fueron $S$. pneumoniae $40 \%$ (30\% resistentes a penicilina), Haemophilus $28 \%$ (10,9\% productores de $\beta$-lactamasa), y Moraxella 4\%. El SD medio disminuyó de 10 en el ingreso a 3 en el día 3 , y a 1 en el día 10 ( $p<0,001)$. Al día 10 y 40 respectivamente, 48,1 y $74,1 \%$ estaban curados, 45,1 y $18,5 \%$ mejoraron y en 6,8 y $3,1 \%$ había fracasado el tratamiento. La diarrea aumentó de $6,4 \%$ al ingreso a $32,5 \%$ en el día 3 y $8,1 \%$ en el día 10, requiriendo sólo manejo dietético. Dos niños discontinuaron el tratamiento debido a vómitos uno y a exantema el otro. Conclusiones: Amoxicilina/ sulbactam en dosis de 100/25 mg/kg/día durante 10 días tuvo una eficacia clínica (curación o mejoría) de $93,2 \%$. La diarrea transitoria fue común aunque no severa requiriendo sólo restricción dietética.

\section{Bibliografía}

1.- Bluestone C D y Klein J O. Capítulo: Epidemiología. En Otitis Media en lactantes y niños. Bluestone CD y Klein JO eds. $2^{\text {a }}$ edición, 1996, Editorial Médica Panamericana S.A., Buenos Aires. Págs: 39-54.

2.- Rosenblüt A, Santolaya M E, González P, Corvalán V, Avendaño L F, Martínez M A, et al. Bacterial and viral etilogy of acuta otitis media in Chilean children. Pediatr Infect Dis J 2001; 20 (5): 501-7.

3.- American Academy of Pediatrics. Pneumococcal Infections. In Pickering LK, ed. 2000 Red Book Report of the Committee on Infectious Diseases. $25^{\text {th }}$ ed. Elk Grove Village, Il. American Academy of Pediatrics; 2000: 452-60.

4.- Dowell S F, Butler J C, Giebink S, Jacobs M R, Jernigan D, Musher D M, Rakowsky A, Schwartz B, and The Drug Resistant Streptococcus pneumoniae Therapeutic Working Group. Pediatr Infect Dis J 1999; 18 (1): 19

5.- Siri M T, Banfi A, Barroihlet G, Harum A, Prado V. Variaciones de la etiología der otitis media agusa en lactantes durante un período de 4 años de estudio (1979-1983). Rev Chil de Infect 1984; 2: 77-81

6.- Pinto M E, Emparanza P, Salinas V, Giglio M S. Microbiología de la otitis media aguda en el niño. Libro de Resúmenes VII Congreso Chileno de Infectología, Santiago, 9-11 Octubre 1990, p 15.

7.- Mc Cracken Jr G H. Prescribing antimicrobial agents for treatment of otitis media. Pediatr Infect Dis J 1999; 18 (12) 1141-6.

8.- Blumer J L. Fundamental basis for rational therapeutics in acute otitis media. Pediatr Infect Dis J 1999; 18 (12): 1130-40. 
9.- Heitmann I. Epidemiología nacional de las infecciones causadas por Streptococcus pneumoniae. Rev Chil Infect 1999; 16: 13-6.

10.- Ulloa M T, Castillo L, Maldonado A. Estudio de la sensibilidad de Haemophilus influenzae a amoxicilinaácido clavulánico. Rev Chil Infect 1992; 3: 200-4.

11.- Trucco O, Prado V, Mollinedo E, Cordovez A. Actividad de ampicilina-sulbactam versus ampicilina. Rev Méd Chile 1989; 117: 145-8.

12.- Canafax D M, Yuan Z, Chonmaitree T, Deka K, Russlie H Q, Giebink G S. Amoxicillin middle ear fluid penetration and pharmacokinetics in children with acute otitis media. Pediatr Infect Dis J 1998; 17 (2): 149-56.

13.- Seikel K, Shelton S, McCracken G H. Middle ear fluid concentrations of amoxicillin after larger dosages in children with acute otitis media. Pediatr Infect Dis J 1997; 16: 710-1.

14.- Blumer J L. Implications of pharmacokinetics in making choices for the management of acute otitis media. Pediatr Infect Dis J 1998; 17: 565-70.

15.- Bartlett J G. Antibiotic-associated diarrhea. En Remington JA \& Swartz MN eds. Current Clinical Topics in Infectious Disease. Mc Graw-Hill, New York, NY, 1980; pp: 240-64.

16.- Dagan R, Hobermann A, Johnson C, Leibovitz E L, Arguedas A, Rose F V, et al. Bacteriological and clinical efficacy of high dose amoxicillin/clavulanate in children with otitis media. Pediatr Infect Dis J 2001; 20 (9): 829-37.

17.- American Academy of Pediatrics. Table 4.2 Antibacterial drugs for pediatric patients beyond the newborn period. En Pickering LK, ed. 2003 Red Book Report of the Committee on Infectious Diseases. $25^{\text {th }}$ ed. Elk Grove Village, Il. American Academy of Pediatrics; 2003: 702-12.

18.- Craig W A, Andes D. Pharmacokinetics and pharmacodynamics of antibiotics in otitis media. Pediatr Infect Dis J 1996; 15: 255-9.

19.- Jacobs M R. Increasing antibiotic resistance among otitis media pathogens and their susceptibility to oral agents based on pharmacodynamic parameters. Pediatr Infect Dis J 2000; 19(5): S47-56.

20.- Dagan R, Johnson C, McLinn S, Abughali N, Feris J, Leibowits E, et al. Bacteriological and clinical efficacy of amoxicillin/clavulanate vs. azithromycin in acute otitis media. Pediatr Infect Dis J 2000; 19 (2): 95104.

21.- Beam T R, Gilbert D, Kunin C M. Guidelines for the evaluation of anti-infective drug products. Clin Infect Dis 1992; 15 (Suppl 2): 55-532.

22.- Dagan R, Leibovitz E, Greenberg D, Yagupsky P, Fliss D M, Leiberman A. Early eradication of pathogens from middle ear fluid during antibiotic treatment of acute otitis media is associated with improved clinical outcome. Pediatr Infect Dis J 1998; 17 (9): 776-82.

23.- Wong D, Baker C. Pain in children: comparison of assessment scales. Pediatr Nurs 1988; 14 (1): 9-17.

24.- Viada J, Sáenz M, Bravo J P. Experiencia con el uso de una solución de prilocaína y lidocaína (EMLA®) como anestésico en procedimientos otológicos ambulatorios. Rev Chil Otorrinol Cir Cabeza Cuello 1998; 58: 141-6.

25.- National Committee for Clinical Laboratory Standards (NCCLS). Performance standards for antimicrobial susceptibility testing, $10^{\text {th }}$ ed. NCCLS document M100S10. Wayne, PA: NCCLS, 2000.

26.- National Committee for Clinical Laboratory Standards (NCCLS). Performance standards for antimicrobial susceptibility testing, $12^{\text {th }}$ ed. NCCLS document M100-
S12. Wayne, PA: NCCLS, 2002.

27.- Dagan R, McCracken Jr G H. Flaws in design and conduct of clinical trials in acute otitis media. Pediatr Infect Dis J 2002; 21 (12): 894-902.

28.- Jacobs M R, Koeth L M, Appelbaum P C. Use of appropiate breakpoints in antimicrobial surveillance studies. Clin Infect Dis 2002, ; 35 (1Dec): 1446-8.

29.- Lagos R, Muñoz A, Valenzuela M T, Heitmann I, Levine M. Population-based surveillance for hospitalized and ambulatory pediatric invasive pneumococcal disease in Santiago, Chile. Pediatr Infect Dis J 2002; 21 (12): 1115-23.

30.- Bluestone C D, Klein J O. Capítulo: Tratamiento. En: Otitis Media en lactantes y niños. Bluestone CD y Klein JO, eds. $2^{a}$ edición, 1996, Editorial Médica Panamericana S.A., Buenos Aires. Págs: 145-241.

31.- Howie V M, Ploussard J H. The "in vivo sensitivity test": bacteriology of middle ear exudate. Pediatrics 1969; 44: 940-4.

32.- Howie V M. Natural history of otitis media. Ann Otol Rhinol Laryngol 1975; 84 (Suppl 19): 67.

33.- Klein J. The "in vivo sensitivity test" for acute otitis media revisited. Pediatr Infect Dis J 1998; 17((9): 774-5.

34.- Klein J O. Microbiologic efficacy of antibacterial drugs for acute otitis media. Pediatr Infect Dis J 1993; 12: 973-5.

35.- Hoberman A, Paradise J L, Block S, Burch D J, Jacobs M R, Balanescu M I. Efficacy of amoxicllin/clavulanate for acute otitis media: relation to Streptococcus pneumoniae susceptibility. Pediatr Infect Dis J 1996; 15 (10): 955-62.

36.- Chonmaitree T, Owen M J, Howie V M. Respiratory viruses interfere with bacteriological response to antibiotic in children with acute otitis media. J Infect Dis 1990; 162: 548-9.

37.- Arola M, Ruuskanen O, Ziegler T, Mertsola J, NantoSalonen K, Putto-Laurilo A, et al. Clinical role of respiratory virus infection in acute otitis media. Pediatrics 1990; 86 (6): 548-55.

38.- Mansilla E, Vinent P, Chalup M, Sturla S. Efectividad de amoxicilina-sulbactam administrada cada 12 horas en niños con otitis media aguda. VII Congreso Latinoamericano de Infectología Pediátrica y III Congreso Venezolano de Infectología "Dr. Raúl Isturiz", Caracas, Venezuela, Noviembre, 1997.

39.- Desio E, López Mina S, Meder O, Páez R, Salinas A, Zanzi V. Actividad terapéutica de la asociación amoxicilina-sulbactam administrada cada 12 horas en niños con otitis media aguda. Estudio multicéntrico. 1999 (Data on file).

40.- Högenauer C, Hammer H, Krejs G, Reisinger E. Mechanisms and management of antibiotic-associated diarrhea. Clin Infect Dis 1998; 27: 702-10.

41.- McLinn S E, Moskal M, Goldfarb J, Bodor F, Aronovitz G, Schwartz R, et al. Comparison of cefuroxime axetil and amoxicillin-clavulanate suspensions in treatment of acute otitis media with effusion in children. Antimicrob Agents Chemother 1994; 38 (10): 2516-9.

42.- Manford Gooch III W, Blair E, Puopolo A, Paster Z, Schwartz R H, Miller C, et al. Clinical comparison of cefuroxime axetil and amoxicillin/clavulanate suspension in the treatment of pediatric patients with acute otitis media with effusion. Clin Ther 1995; 17 (5): 838-51.

Correspondencia a:

José Cofré Guerra

E-mail: pepecofre@123.cl 